\title{
Cavity preparation/assembly techniques and impact on $Q$, realistic $Q$-factors in a module, review of modules
}

\author{
Peter Kneisel* $^{*}$ \\ Jefferson Laboratory, 12000 Jefferson Avenue, Newport News, VA 23606, USA
}

Available online 22 November 2005

\begin{abstract}
This contribution summarizes the surface preparation procedures for niobium cavities presently used both in laboratory experiments and for modules, such as buffered chemical polishing (BCP), electropolishing (EP), high pressure ultrapure water rinsing (HPR), $\mathrm{CO}_{2}$ snow cleaning and high temperature heat treatments for hydrogen degassing or post-purification. The impact of surface treatments and the degree of cleanliness during assembly procedures on cavity performance ( $Q$-value and accelerating gradient $\left.E_{\text {acc }}\right)$ will be discussed.

In addition, an attempt will be made to summarize the experiences made in module assemblies in different labs/projects such as DESY (TTF), Jlab (Upgrade) and SNS.
\end{abstract}

(C) 2005 Elsevier B.V. All rights reserved.

PACS: 29.27.Bd, 41.60.Cr

Keywords: Radio frequency; Superconductivity; Surface treatment; Niobium; Cryo modules

\section{Introduction}

Superconducting RF technology has become the "darling" of presently executed (SNS) and newly proposed accelerator projects. These include applications such as energy recovery linacs (ERL), free electron lasers (FEL), proton drivers, accelerators for transmutation of nuclear waste and most recently the recommendation by the International Technology Recommendation Panel (ITRP) for the International Linear Collider (ILC). The reasons for this popularity are astonishing advances in the technology over the last few years, promising better performances of the proposed devices and reduced capital and operational costs. In some instances like e.g. cw machines with high accelerating gradients such as ERL's this technology seems to be the only choice for verifying such designs.

The technology has come a long way since its early application four decades ago at Stanford University [1]. Many technological obstacles such as multipacting, early

\footnotetext{
*Tel.: + 17572697646 ; fax: + 17572697658 .

E-mail address: kneisel@jlab.org.
}

quenches due to defects in the material, high residual resistances (low $Q$-values) or excessive non-resonant electron loading ("field emission") due to contaminated surfaces had to be overcome to arrive at "present-day" performance levels in niobium cavities - the material of choice for accelerator application-which in some instances have been close or even have reached the fundamental limits of the superconducting material. Key to these advances has been a better understanding of material properties, both surface and bulk properties, and physical phenomena caused by the interplay between electro-magnetic fields in the cavities and the state of the cavity surfaces. Also, improved procedures to protect the sensitive cavity surfaces from contamination - after all in RF superconductivity only a few nanometer of the material are determining the performance of a cavity-have contributed significantly to the nowadays achievable performance levels of cavities.

In the following sections (2-4), established and developing techniques for preparation of high performance RF cavity surfaces are being reviewed along with assembly techniques to preserve the necessary cleanliness levels and to avoid re-contamination. 
In Section 5 the performances of cryo-modules in largescale projects such as SNS, TTF/DESY and Jlab's Upgrade are reviewed.

\section{2. "Standard" cavity surface treatments}

The performance of an accelerator cavity is determined by the dependence of its $Q$-value on accelerating gradient. The goal is to achieve a performance as close as possible to the theoretical limit of the superconducting material. The $Q$-value, which is inversely proportional to the surface resistance, is given by the BCS theory and the achievable accelerating gradient is determined by the associated peak magnetic field on the cavity surface, at which the superconductor is transitioning into the normal conducting state - the critical magnetic field, approximately $185 \mathrm{mT}$ for niobium at $2 \mathrm{~K}$. As an example, Fig. 1 shows the frequency dependence of the BCS surface resistance for niobium at a temperature of $2 \mathrm{~K}$, which is in many cases the operating temperature for cavities of a frequency above $\sim 700 \mathrm{MHz}$. However, the BCS theory describes the ideal surface resistance at low surface fields; realistically, there is not only an additive contribution to the BCS behaviorthe residual resistance $R_{\text {res }}$ - but also a field dependence as schematically shown in Fig. 2. At the high end of the $Q$ vs. $E$-curve a strong degradation of the $Q$-value is often observed. The physics of this " $Q$-drop" is still not completely understood, however it has become clear that oxygen diffusion from the surface into the material plays a role, since "in situ" baking of a cavity at $120^{\circ} \mathrm{C}$ for long periods of time $(>24 \mathrm{~h})$ improves the high field $Q$ values [2].

The purpose of the treatment of a niobium cavity surface is to achieve an "ideal" surface as closely as possible.

Niobium cavity surfaces are damaged by the fabrication process, starting from the rolled and annealed sheet material through the forming, machining and electron beam welding processes. In order to achieve good performances of cavities this "damage layer" has to be removed. Fig. 3 shows the results from a series of experiments, during which material has been successively removed from a cavity surface and the residual surface resistance at low fields and the maximal achievable surface electric fields have been determined. It is obvious that at least a layer of $100 \mu \mathrm{m}$ has to be removed to achieve low residual resistances (high $Q$-values) and even more for high gradients [3].

To achieve this "ideal" surface-free of defects, damages, contamination and residue from handling procedures - a series of surface treatment procedures have been developed over the years at various labs involved in SRF technology, which have led to the mature technology available nowadays. The commonly used procedures are listed and discussed below:

- Eddy current scanning and squid scanning of niobium sheets prior to cavity fabrication to detect defects in the material: This QA procedure has been successfully developed at DESY for the 9-cell cavities for the

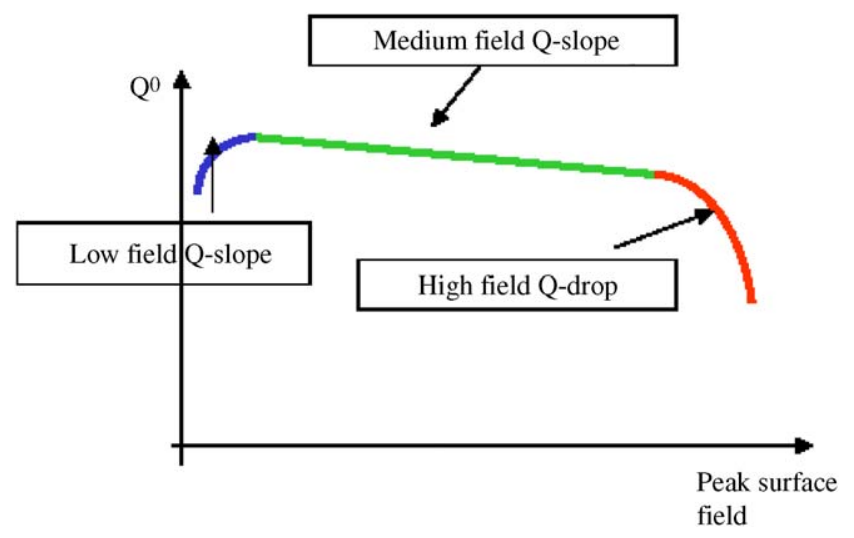

Fig. 2. Schematic of the often observed dependence of $Q$-value on accelerating gradient in high purity niobium cavities.

Frequency Dependence of Rbcs

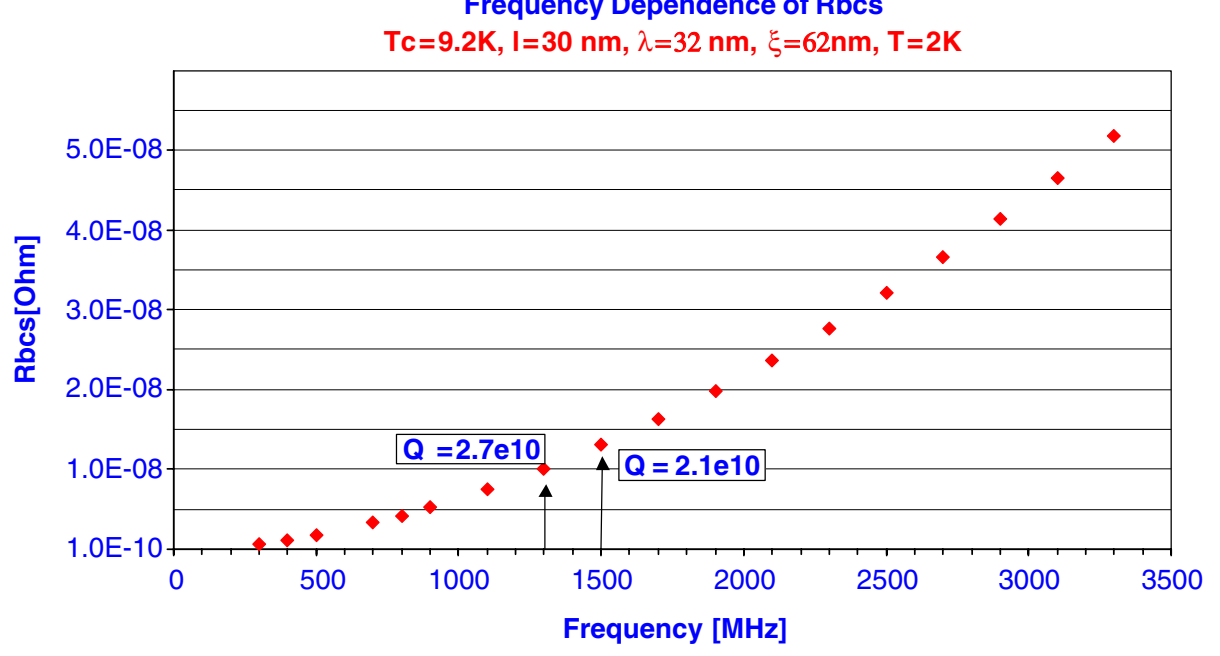

Fig. 1. Frequency dependence of the BCS surface resistance for niobium. 

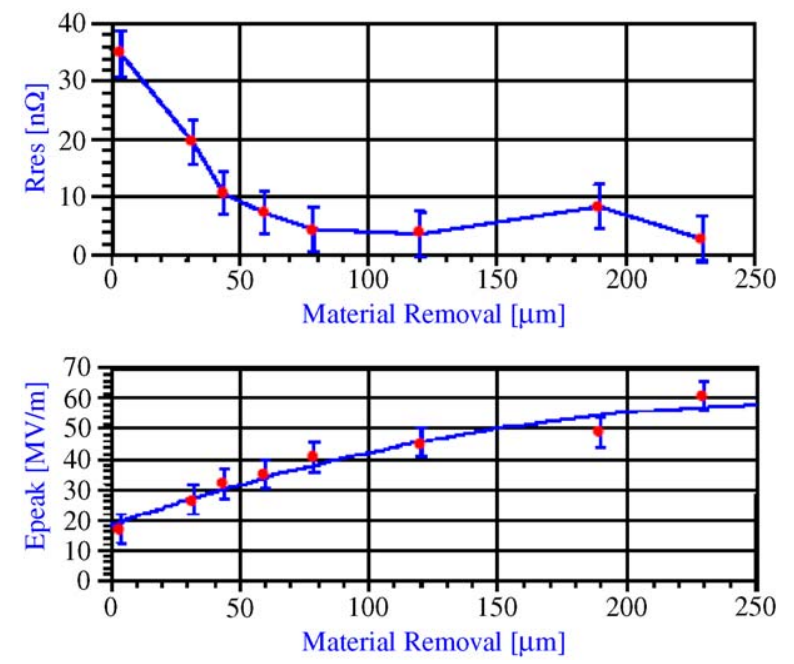

Fig. 3. Improvement of niobium cavity performance by successive removal of the surface damage layer.

TESLA Test Facility (TTF) [4,5]. The resolution for the eddy current system is limited to defects of approximately $100 \mu \mathrm{m}$, whereas the squid system [6] provides a resolution of $<50 \mu \mathrm{m}$.

- Degreasing of the cavities under ultrasonic agitation: this method is routinely used with detergents or solvents to remove greasy or oily residue from fabrication processes and finger prints.

- Buffered chemical polishing to remove the surface damage layer: the solution consists of an acid mixture of hydrofluoric $(40 \%)$, nitric $(69 \%)$ and phosphoric $(86 \%)$ acids of mixing ratios $1: 1: 1,1: 1: 2$ or $1: 1: 4$. The more buffered solutions provide lower removal rates and therefore a better control of the exothermic reaction. The chemical treatment is done at room temperature for smaller cavities; more typical for larger cavities a $1: 1: 2$ mixture at a temperature around $10^{\circ} \mathrm{C}$ is used to avoid excessive pick-up of evolving hydrogen gas from the process [7].

- Electropolishing (EP) is used to remove the surface damage layer and to achieve a very smooth surface finish on the niobium. The electropolishing process for niobium was developed in 1971 [8] with a solution of hydrofluoric and concentrated sulfuric acids in a 1:9 ratio. The process has a leveling effect on the surfaces since higher electric fields are established in the bath at surface protrusions and therefore the removal rate at these sites is higher. The original process controlled the current oscillations occurring during the electropolishing; later the process was improved at KEK to a continuous process [9] for horizontally rotating cavity arrangements. EP is presently used/developed at several laboratories such as KEK, DESY, Saclay, Cornell and Jlab. It has been demonstrated on many occasions (see e.g. Ref. [10]) that superior cavity performances can be achieved with electropolished surfaces.
- "Barrel" polishing with plastic stones, an abrasive powder and water: this process has been developed at KEK to mechanically remove imperfections on the cavity surface resulting from the fabrication process such as surface cracks, rough electron beam welds or weld splatter. The process is rather slow, but by adding an additional motion ("Centrifugal barrel polishing") during the process as schematically indicated in Fig. 4 the removal rate was increased to approximately $4 \mu \mathrm{m} / \mathrm{h}$ [11]. By replacing the water with a hydrogen-free agent such as e.g. FC-77 the niobium was kept hydrogen-free and the need for hydrogen degassing was eliminated, stream-lining the whole surface treatment process [12].

- High Pressure Ultrapure Water Rinsing is universally used as a final step in the surface preparation of a niobium accelerating cavity. Ultrapure water jets of a resistivity $>18 \mathrm{M} \Omega \mathrm{cm}$ are directed at the cavity surfaces at a pressure of $\sim 100$ bar (1200 psi) through spray nozzles made from either sapphire or stainless steel. The surfaces are "scanned" by the water jets either in a continuous mode by rotation of the cavity accompanied with up/down movements of the cavity or the highpressure wand or by single or multiple sweeps of the jets along the rotating cavity. It has been established also that additional high-pressure rinses after the attachment of auxiliary parts such as couplers or valves to the cavity are beneficial to reduce field emission loading [13]. Fig. 5 illustrates the process and shows as an example the High Pressure Rinse cabinet installed at Jlab.
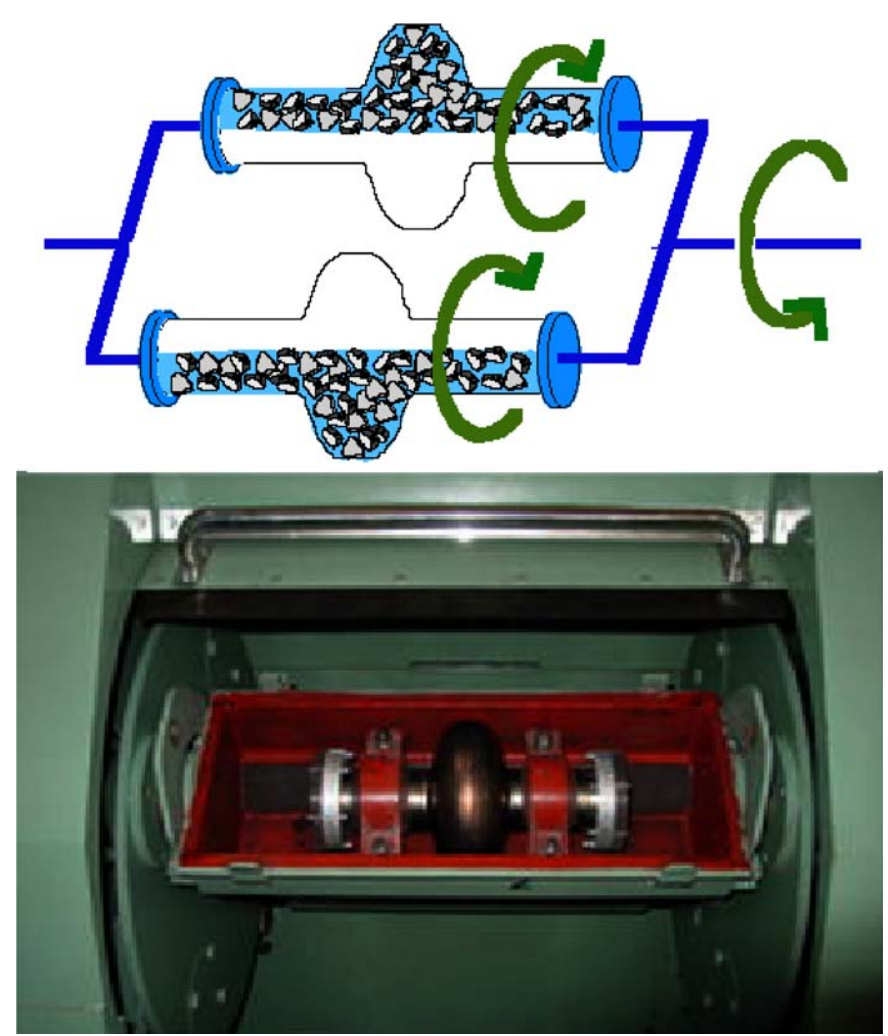

Fig. 4. Centrifugal barrel polishing as developed at KEK. 


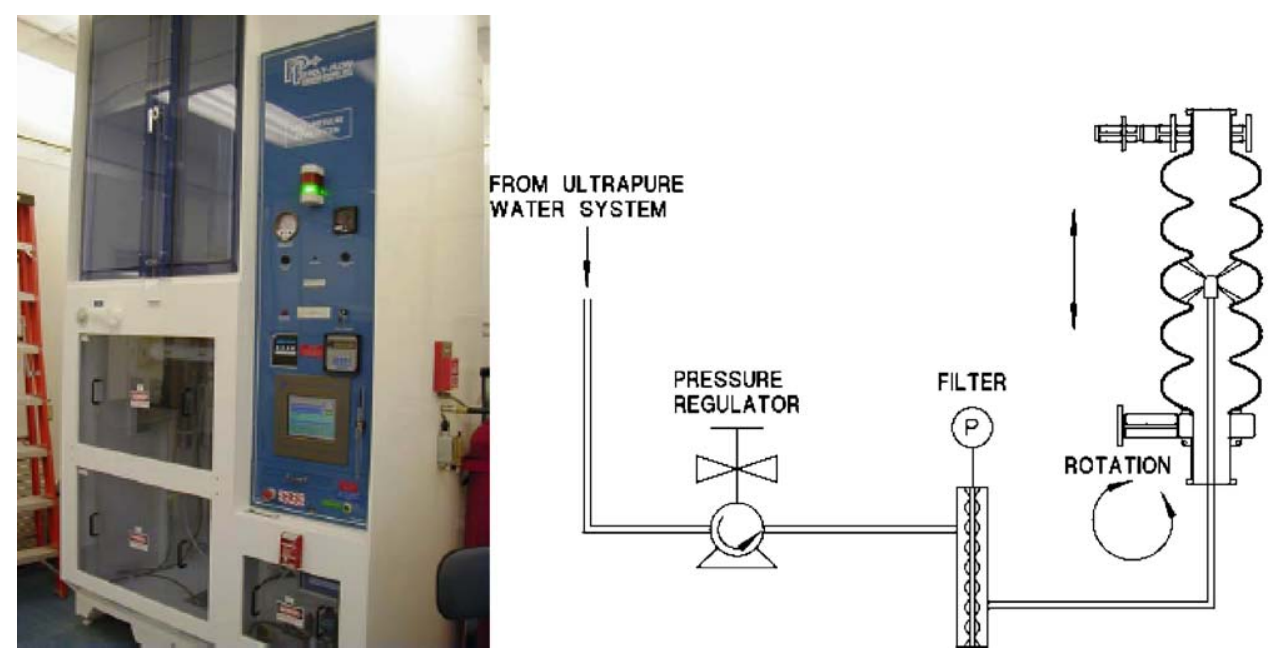

Fig. 5. High pressure ultrapure water rinsing facility at Jlab.

- High Temperature Ultra High Vacuum Heat Treatment is often applied to the niobium cavities with a dual purpose:

$\bigcirc$ degassing of the interstitially dissolved hydrogen from the chemical surface treatment is carried out at temperatures between 600 and $800{ }^{\circ} \mathrm{C}$ for several hours,

$\bigcirc$ post purification of the cavities for improvement of the thermal stability ( increase of RRR-value) is typically done for several hours between $1200^{\circ} \mathrm{C}$ and $1400{ }^{\circ} \mathrm{C}$ in the presence of a solid state getter material such as Ti. The improvement of the thermal stability is accompanied with a degradation of the mechanical properties of the niobium.

- "In situ" baking: as shown in Fig. 2 the high field $Q$ values often degrades by several factors. Improvements of this behavior can be achieved by baking the cavities "in situ" at a temperature of $\sim 120^{\circ} \mathrm{C}$ for $>24 \mathrm{~h}$ [2]; this improvement is often more pronounced for electropolished cavities, but is also observed with cavities which have undergone buffered chemical polishing.

\section{3. "Alternative surface" treatments}

Here we will discuss

- Alternative cleaning methods such as $\mathrm{CO}_{2}$ snow Among the various alternative surface cleaning techniques [14] $\mathrm{CO}_{2}$-snow cleaning is being developed at DESY as an alternative to high pressure water rinsing or as an "in situ" cleaning technique for assembled modules [13]. The method has been applied successfully to samples, which were after cleaning investigated at the University of Wuppertal with respect to their DC field emission behavior [15]. Even though an optimization of the process is necessary (avoidance of condensation, mass flow optimization), preliminary tests on single cell cavities showed encouraging results and gradients up to $E_{\text {acc }}=33 \mathrm{MV} / \mathrm{m}$ were measured.

A production system is presently under construction and will be completed in autumn of 2005 .

- Large grain/single crystal cavities

It has recently been recognized that ingot niobium material with very large grains or single crystals has very good mechanical properties such as elongation and can easily be formed into cavities. In addition, buffered chemical polishing provides on this material very smooth surfaces, which are believed to be necessary for high performance cavities as experienced with electropolished surfaces. Fig. 6 compares the surface roughness of a buffered chemically polished niobium single crystal to a surface of fine grain material [16]. A nearly 50 times smoother surface results from the bcp of a single crystal and compared to an electropolished fine grain surface a gain of about a factor of 10 in smoothness has been measured (RMS roughness of $27 \mathrm{~nm}$ for single crystal vs. $251 \mathrm{~nm}$ of electropolished fine grain niobium).

The RF performance of a single cell single crystal cavity at $2.2 \mathrm{GHz}$ is shown in Fig. 7 together with scaled values for a frequency of $1300 \mathrm{MHz}$, a most likely frequency for an ERL device. This "new" technology seems to provide not only excellent cavity performances, but also promises potential advantages such as reduced cost for the material and the cavities, less elaborate QA and surface treatment procedures and better mechanical properties of the material.

\section{Comparison of TTF module and SNS module assembly procedures}

It is widely accepted that the obstacles to good cavity performance are insufficient material removal, defects in the material and contamination of the 

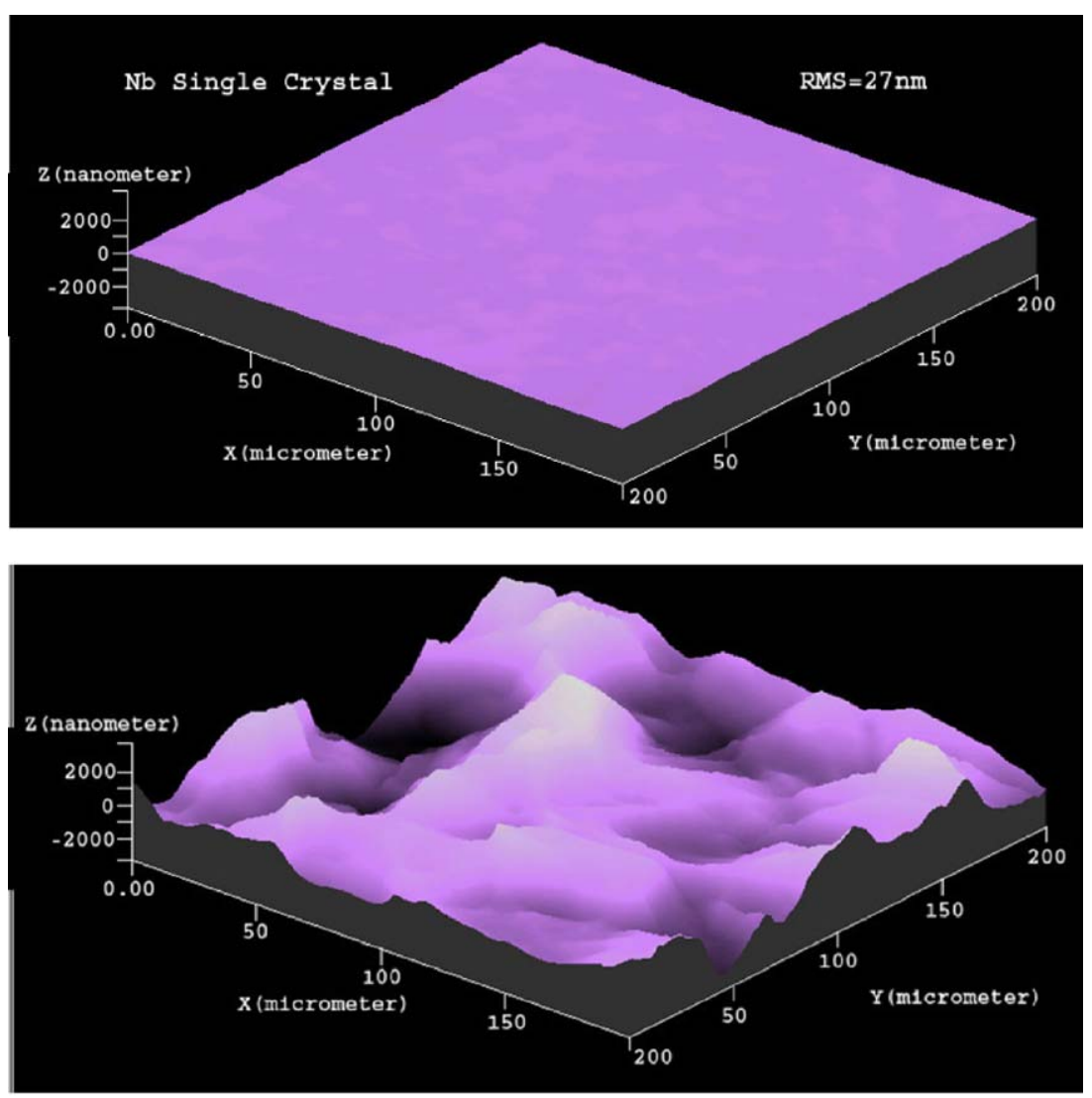

Fig. 6. Comparison of surface roughness achieved with BCP on single crystal and fine grain niobium.

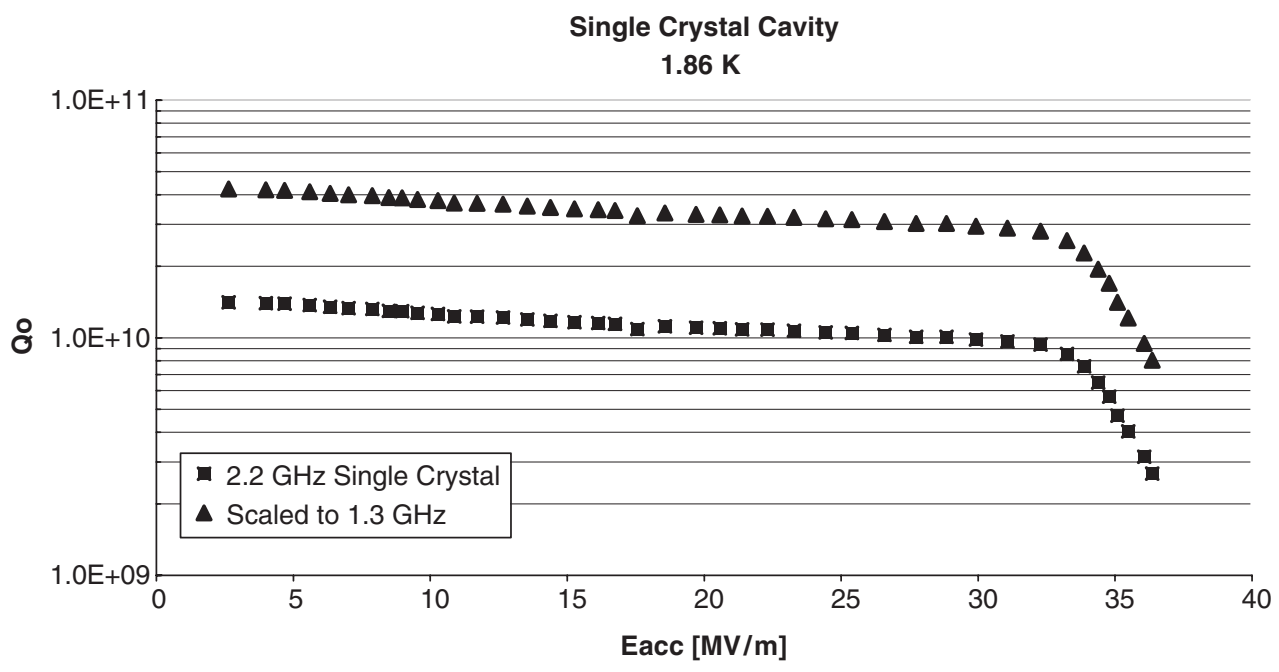

Fig. 7. Performance of a single crystal niobium cavity at $2.2 \mathrm{GHz}$. The $Q$-values are in addition scaled to a frequency of $1.3 \mathrm{GHz}$.

sensitive surfaces (field emission). All applied procedures need to deal with these problems and the most difficult is the control of contamination. The level of contamination is different in the different laboratories and depends on the infra-structure, the design of the cavities/modules, the auxiliary parts to the cavities such as bolts, gaskets, or couplers and most importantly on the people. The optimum procedures have to be developed for each lab and each project. The following comparison as listed in Tables 1 and 2 between procedures applied at DESY to the TTF modules (\# 1-5) [17] and 23 modules for the SNS project at Jlab points out the common procedures, but also the different approaches on various details.

The assembly of a TTF string in the clean room at DESY is shown in Fig. 8. 
Table 1

Cavity treatment and assembly procedures for TTF cryo-modules and SNS/Jlab Modules (the entries are: italic $=$ common, bold $=$ TTF, Comic Sans MS = SNS/Jlab)

Outside bcp (> 20 micron), inside bcp ( 80 - 100 micron), Ultra Pure Water ( UPW)rinsing

Hydrogen degassing ( $600^{\circ} \mathrm{C}$ for SNS, $800^{\circ} \mathrm{C}$ for TTF )

Rinsing in Ultra Pure Water, post purification with Ti at $1400^{\circ} \mathrm{C}$

BCP to remove Ti layer : 80 micron inside, 40 micron outside, Ultra Pure Water rinsing

Re - tuning

\begin{tabular}{|l|l}
\hline 20 micron bep inside, UPW rinse & No VTA test of bare cavity for SNS
\end{tabular}

High pressure rinse, $12 \mathrm{hrs}$ drying in class 10

Assembly of auxiliary parts

Vacuum leak check of flange connections

Venting, dismounting of pumping flange

1. + 2. high pressure rinse ( check of \# of particles, TOC..), drying in class 10 Installation of antenna for VTA test

Vertical test

Helium vessel welding by TIG (inert gas inside cavity) or EBW

Preparation for Vertical test or Horizontal test in Chechia

\begin{tabular}{|l|l|}
\hline \multicolumn{1}{|c|}{ Horizontal Test } & Vertical Test \\
\hline Disassembly, degreasing & Re-tuning, degreasing \\
\hline Inside bcp 20 micron, high pressure rinse & $\sim 50$ micron bcp, UPW rinse \\
\hline Drying in class 10 & 2 passes of high pressure rinse \\
\hline Assembly of auxiliary parts & Drying in class 10 for $12 \mathrm{hrs}$ \\
\hline Leak check & Assembly of auxiliary parts \\
\hline $\begin{array}{l}\text { 1. + 2. high pressure rinse ( check of \# of } \\
\text { particles, TOC..), drying in class 10 }\end{array}$ & 2 passes of high pressure rinse \\
\hline Assembly of final flange & Final flange/pump-out port assembly \\
\hline Evacuation, leak checking, venting & Evacuation and leak check \\
\hline $\begin{array}{l}\text { Assembly of power coupler ( avoids } \\
\text { loosing conditioning effect) }\end{array}$ & $\begin{array}{l}\text { Cavity hermetically sealed on test } \\
\text { stand }\end{array}$ \\
\hline Horizontal test & Vertical test at 2K \\
\hline
\end{tabular}


Table 2

Cavity string assembly procedures for TTF modules and SNS modules after qualification of cavities with helium vessels

\begin{tabular}{|l|l|}
\hline Disassembly from horizontal cryostat & $\begin{array}{l}\text { After VTA test (without Higher } \\
\text { Order Mode (HOM) probes and } \\
\text { fundamental power coupler (FPC)): }\end{array}$ \\
\hline $\begin{array}{l}\text { Cleaning for string assembly in class 10 } \\
\text { clean room( "dirty" to class 10000 to } \\
\text { class 10) }\end{array}$ & Disassembly, degreasing \\
\hline Venting of cavity in class 10 & $\begin{array}{l}\text { Additional 10 -20 micron bcp, UPW } \\
\text { rinse }\end{array}$ \\
\hline Assembly of gate velves, magnets.. & $\begin{array}{l}\text { High Pressure Rinse for } 4 \mathrm{hrs,} \\
\text { drying in class } 10 \text { for } 12 \mathrm{hrs}\end{array}$ \\
\hline $\begin{array}{l}\text { "on the job" cleaning of bolted beam } \\
\text { pipe flanges }\end{array}$ & Attachment of HOM probes \\
\hline Final leak check & $\begin{array}{l}\text { Additional } 4 \text { hrs of high pressure } \\
\text { rinsing, drying in class 10 }\end{array}$ \\
\hline $\begin{array}{l}\text { Venting for transportation to } \\
\text { installation in cryostat }\end{array}$ & $\begin{array}{l}\text { Assembly on assembly rail with FPC, } \\
\text { bellows and gate valves, beam pipe } \\
\text { opening closed with Niobium plate }\end{array}$ \\
\hline Installation into cryostat & $\begin{array}{l}\text { Assembly of string takes several } \\
\text { days for the } 4 \text { cavities }\end{array}$ \\
\hline & $\begin{array}{l}\text { Final string assembly completed } \\
\text { with connecting beam line bellows }\end{array}$ \\
\hline & $\begin{array}{l}\text { Evacuation with turbo pump and leak } \\
\text { checking }\end{array}$ \\
\hline $\begin{array}{l}\text { Transfer to cryo-module assembly } \\
\text { area and installation in cryostat }\end{array}$ \\
\hline
\end{tabular}

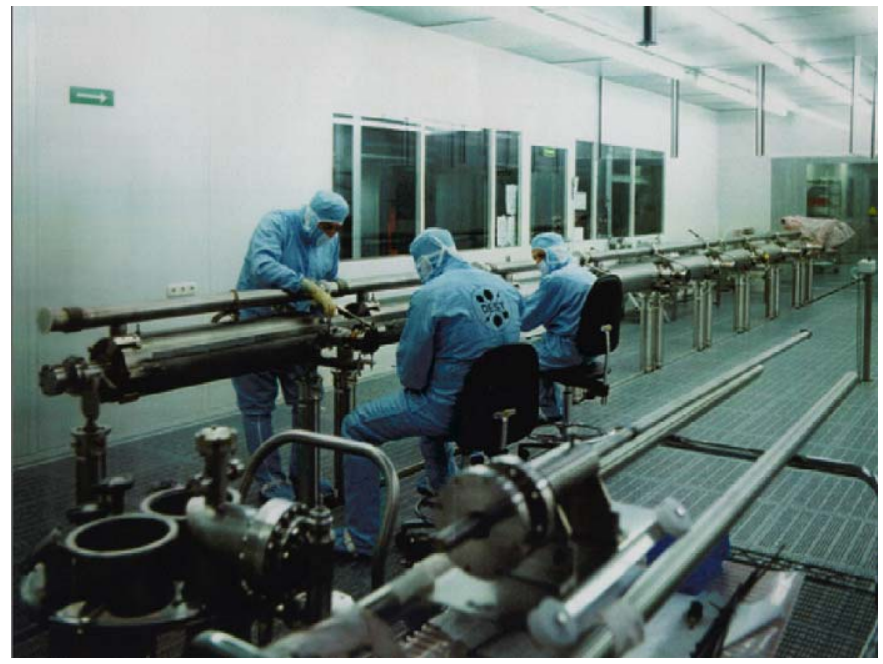

Fig. 8. Assembly of a TTF cavity string in the class 10 clean room at DESY.

\section{Cryomodule performances}

Whereas many data are available for individual cavity performances, cryo-modules on a larger scale have only been tested in recent years for the TTF-, SNS- and Jlab Upgrade projects.

\subsection{TTF}

Approximately 80 nine-cell cavities were produced by three suppliers in three production runs over a many year span. Gradients and their spread improved significantly; nine modules with 8 cavities each were assembled, making use of 58 different cavities, all treated by BCP. In the modules the average gradients improved from $18.7 \mathrm{MV} / \mathrm{m}$ for the first module to $26 \mathrm{MV} / \mathrm{m}$ for the third. Meanwhile electropolishing was developed in collaboration with KEK 
and significant improvements in gradients were realized. The average value improved from $\sim 29 \mathrm{MV} / \mathrm{m}$ for standard $\mathrm{BCP}$ [18] to $35.6 \mathrm{MV} / \mathrm{m}$ for electropolished cavities [19]. Fig. 9 shows results from vertical tests of several electropolished cavities. One of these cavities (AC 72) was installed field emission-free into an accelerating module for the VUV-FEL without degradation of performance up to $35 \mathrm{MV} / \mathrm{m}$. Electropolishing becomes the Stateof-the-Art surface preparation technique for the X-FEL installation at DESY.

\subsection{SNS}

Between the year 2002 and 2005 eleven medium beta cryomodules with three cavities each and 12 high beta cryomodules with four cavities each have been assembled and tested at Jlab for the SNS project. The cavities were fabricated in accordance with Jlab's specifications by ACCEL Instruments and qualified at Jlab for installation into modules. All cavities performed to the specified values for $Q$-value and accelerating gradient after initial problems with the infra-structure at Jlab had been overcome and the procedures as listed in Tables 1 and 2 had been established. However, control of contamination and therefore the onset of field emission remained a re-occurring concern. In general, no significant differences in cavity performance were found between vertical tests (cw test) and tests of the cavities in the modules (pulsed test) [20].

In some cases better performances were achieved in the module tests, which allowed easier RF processing due to the availability of large input power. Fig. 10 is a summary plot of the gradients at the specified $Q$-value of $5 \times 10^{9}$ measured over a 2 year span between October 2002 and October 2004; it shows both some "seasonal" variation in performance and a rather wide spread,

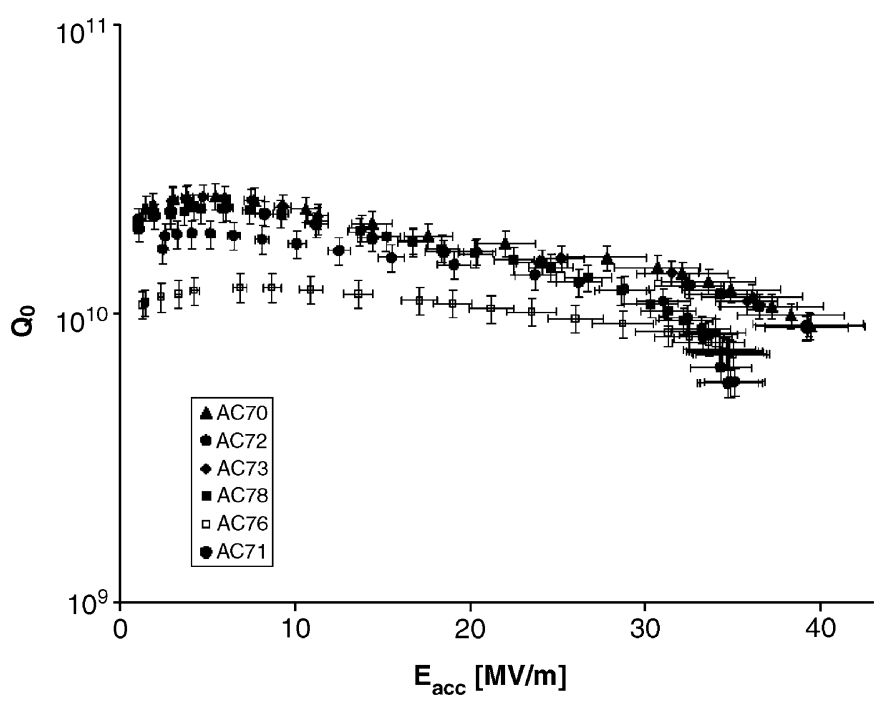

Fig. 9. Experimental results achieved on 9-cell TTF cavities during vertical testing after electropolishing surface treatment.

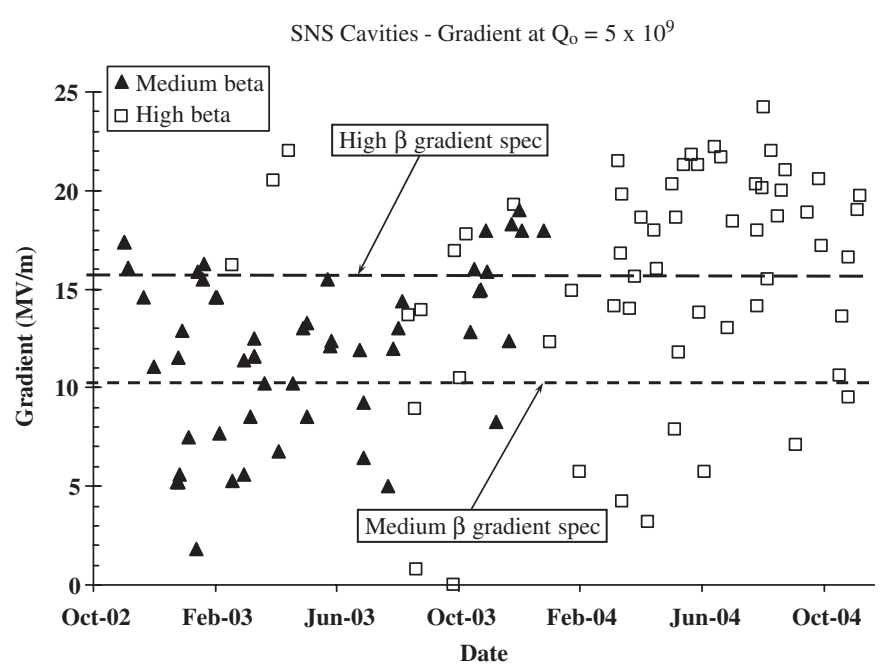

Fig. 10. Summary of results from SNS cavity tests during October 2002 and October 2004.

\section{LL Cavities for Renascence - VTA Performance}

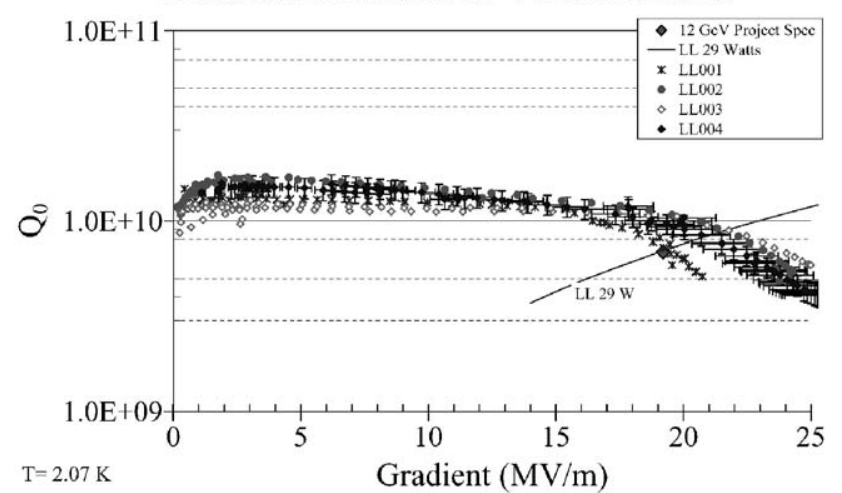

Fig. 11. Vertical test performance of 7-cell cavities for the "Renascence" cryomodule at Jlab.

indicative of some QA and infra-structure problems with the facilities at Jlab.

\subsection{Jlab upgrade}

At Jlab three different modules have been developed since the initial installation of CEBAF.

Two of these modules were used for the FEL with the modification of the original cavity design from five cell cavities to 7-cell cavities and a replacement of the waveguide HOM couplers with coaxial-DESY type HOM couplers [21]. The cavities are stably operated at $E_{\text {acc }}=15 \mathrm{MV} / \mathrm{m}$ in the FEL [22].

A new module, named "Renascence", has been developed with two different 7-cell cavity geometries: a high gradient (HG) cavity optimized for accelerating gradient and a low loss (LL) cavity minimizing the cryogenic losses [23]. The design goals for these cavities in a module are a gradient of $\sim 20 \mathrm{MV} / \mathrm{m}$ with a $Q$-value at $2.05 \mathrm{~K}$ of $Q=8 \times 10^{9}$. Several cavities of each geometry have been fabricated "in house" at Jlab and have been qualified in vertical tests [24]. Results are shown in Fig. 11. The cavities 
have been assembled into a cavity string and the assembly into the cryostat is nearly complete. Test of the module is expected to take place in summer 2005.

\section{Conclusion}

The preparation and assembly of niobium multi-cell cavities into cavity strings/cryomodules has matured to a point, where one can achieve accelerating gradients $E_{\text {acc }}>15 \mathrm{MV} / \mathrm{m}$ with $Q$-values of $Q>8 \times 10^{9}$ at $2 \mathrm{~K}$. However, field emission is still the major obstacle to achieve these performance levels on a regular basis and stringent contamination control measures have to be applied. At gradients above $20-24 \mathrm{MV} / \mathrm{m}$ a not yet fully understood " $Q$-drop" is encountered, which can be overcome - in most cases - by "in-situ" baking at $\sim 120^{\circ} \mathrm{C}$ for many hours. This improvement seems to be more consistent on electropolished cavities, but it is also observed with buffered chemically polished surfaces.

Finally, as already pointed out above, the optimum procedures are facility and project specific and cavity performances seem to be "local", depending a lot on well trained personnel.

\section{Acknowledgements}

Many colleagues have provided me with information for this contribution and I used figures from presentations by several colleagues, whom I want to thank for their support: C. Reece, Jlab, J. Ozelis, Jlab, H. Whitehead, Jlab, A. Matheisen, DESY, L. Lilje, DESY, H. Weise, DESY, W. Singer, DESY and D. Reschke, DESY.

\section{References}

[1] H.A. Schwettman, et al., International. Advances in Cryogenic Engineering, Plenum Press, New York, 1965, p.88.
[2] B. Visentin, Proceedings of the 11th Workshop on RF Superconductivity, Travemuende, Germany (2003) paper TuO01, https:// srf2003.desy.de/sl-srf/

[3] P. Kneisel, B. Lewis, Proceedings of the 7th Workshop on RF Superconductivity, Gif-sur-Yvette, France, 1995, p. 311.

[4] W. Singer, D. Proch, A. Brinkmann, Proceedings of the Eighth Workshop on RF Superconductivity, Abano Terme, Italy, 1997, p. 850 .

[5] W. Singer, http://www-bd.fnal.gov/niobium/program.html

[6] http://www-mhf.desy.de/public/care04/2004.11.03_sr3/wp6_wuppertal_singer_palmieri.pdf.

[7] B. Bonin, R.W. Roeth, Proceedings of the Fifth Workshop on RF Superconductivity, Hamburg, Germany, 1991, p. 210.

[8] H. Diepers, et al., Phys. Lett. 37A (2) (1971) 139.

[9] K. Saito et al., Proceedings of the Fourth Workshop on RF Superconductivity, KEK, Tsukuba, Japan, 1989.

[10] K. Saito; http://accelconf.web.cern.ch/accelconf/p03/PAPERS/ ROAA002.PDF

[11] T. Higuchi et al., http://conference.kek.jp/SRF2001, paper PR022

[12] T. Higuchi, K. Saito, https://srf2003.desy.de, paper We015

[13] D. Reschke; ANL Report 05/10, Argonne National Lab, 2005, p. 30.

[14] P. Kneisel, B. Lewis, Proceedings of the Seventh Workshop on RF Superconductivity, Gif-sur-Yvette, France, 1995, p. 311.

[15] L. Lilje et al.; http://www.mhf.desy.de/public/care04/2004.11.03_sr3/ wp5_summary_11_200410.pdf

[16] A. $\overline{\mathrm{W}} \mathrm{u}, \mathrm{Jlab}$, private communications

[17] A. Matheisen, http://lcdev.kek.jp/ILCWS/Talks/13wg5-02-FabProc_Matheisen.pdf

[18] H. Weise; http://adweb.desy.de/ weise/:

[19] L. Lilje, http://bel.gsi.de/linac2004/PAPERS/WE102.PDF

[20] J. Ozelis, Jlab, private communications

[21] J. Delayen, Proceedings of the Ninth Workshop on RF Superconductivity, Santa Fe, New Mexico, Paper TuP019, 1999.

[22] A.M. Valente et al., http://accelconf.web.cern.ch/accelconf/e04, paper TUPKF 072

[23] J. Sekutowicz et al., http://accelconf.web.cern.ch/accelconf/p03, paper TPAB 085

[24] C. Reece, ANL Report 05/10, Argonne National Lab, 2005, p. 210 . 